\title{
ON THE SCHRÖDINGER EQUATIONS WITH A NONLINEARITY IN THE CRITICAL GROWTH
}

\author{
JIAN ZHANG
}

\begin{abstract}
In this paper, we consider the Schrödinger equation with a nonlinearity in the critical growth. The purpose of this paper is to establish the existence of ground states via variational methods.
\end{abstract}

\section{Introduction}

In this paper, we consider the following problem:

$$
-\Delta u+V(x) u=a(x) f(u)+u^{2^{*}-1}, \quad u \in H^{1}\left(\mathbb{R}^{N}\right),
$$

where $N \geq 3,2^{*}=2 N /(N-2)$. We are interested in the existence of ground state solutions. Recall that $u$ is said to be a ground state solution of (1.1) if and only if $u$ solves (1.1) and minimizes the functional associated to (1.1) among all possible nontrivial solutions.

This equation or the more general one

$$
-\Delta u=h(x, u), \quad u \in H^{1}\left(\mathbb{R}^{N}\right)
$$

arises in various branches of mathematical physics and it has been studied under various assumptions on $h$. When $h(x, u)=h(u)$ is of subcritical growth, almost necessary and sufficient conditions for the existence of ground state solutions

2010 Mathematics Subject Classification. 35J60, 35A15.

Key words and phrases. Schrödinger equations, critical growth, variational method.

This project is supported by National Science Foundation of China (Grant No. 11401583), the Fundamental Reasearch Funds for the central universities (14CX02153A) and ZR2013AM009. 
to (1.2) are given by Berestycki and Lions [8] for $N \geq 3$ and Berestycki et al. [7] for $N=2$. Subsequently, in [1], [29], [31], the authors attempt to complete the study made in [7], [8] by considering nonlinearities with critical growth. When $h(x, u)=-V(x) u+f(x, u)$ is of subcritical growth, the existence of ground state solutions is considered in [18] for an asymptotically linear problem, in [19] for a nonlinearity satisfying conditions introduced by Berestycki and Lions [8] and in [20] under a more natural super-quadratic condition than the AmbrosettiRabinowitz condition. For other related results, we refer the reader to [2], [14], [17], [21], [22], [25] for the subcritical case and [11], [12], [13], [23] for the critical case. When $h(x, u)=-(1+\mu V(x))+f(u)$ with $\mu>0$, the existence, multiplicity and concentration of solutions are also discussed. See for example [4], [5], [6], [10], [16], [26], [27] and the references therein.

Note that when $h(x, u)$ is of critical growth and depending on $x$ non-radially, only a few results are known for the existence of ground state solutions. Recently, in [30], the authors considered the case of $h(x, u)=-V(x) u+f(u)$ and established a Berestycki-Lions Theorem in the critical case. In this paper, we continue to solve the problem and consider the case of $h(x, u)=-V(x) u+$ $a(x) f(u)+u^{2^{*}-1}$. The functions $V(x), a(x)$ and $f(t)$ are assumed to satisfy some of the following hypotheses:

$\left(\mathrm{V}_{1}\right) V(x) \in C\left(\mathbb{R}^{N}, \mathbb{R}\right), V(x) \geq 0$ and $\lim _{|x| \rightarrow \infty} V(x)=V_{\infty}>0$;

$\left(\mathrm{V}_{2}\right)$ there exist $C_{1}>0$ and $b>0$ such that $V(x) \leq V_{\infty}-C_{1} e^{-b|x|}$ for all $x \in \mathbb{R}^{N}$

$\left(\mathrm{V}_{3}\right) V(x)-V_{\infty} \in L^{N / 2}\left(\mathbb{R}^{N}\right)$

$\left(\mathrm{a}_{1}\right) a(x) \in C\left(\mathbb{R}^{N}, \mathbb{R}\right), a(x) \geq 0$ and $\lim _{|x| \rightarrow \infty} a(x)=a_{\infty}>0$;

$\left(\mathrm{a}_{2}\right)$ there exist $C_{2}>0$ and $a>0$ such that $a_{\infty}-C_{2} e^{-a|x|} \leq a(x) \leq a_{\infty}$ for all $x \in \mathbb{R}^{N}$

(a $\left.a_{3}\right) a(x) \geq a_{\infty}$ for all $x \in \mathbb{R}^{N}$;

$\left(\mathrm{f}_{1}\right) f \in C(\mathbb{R}, \mathbb{R})$ is odd;

(f $\left.f_{2}\right) f(t)=o(t)$ as $t \rightarrow 0_{+}$;

(f $\left.f_{3}\right) \lim _{t \rightarrow+\infty} f(t) / t^{2^{*}-1}=0$;

$\left(\mathrm{f}_{4}\right)$ there exist $D>0$ and $q \in\left(2,2^{*}\right)$ such that $f(t) \geq D t^{q-1}$ for $t \geq 0$;

$\left(f_{5}\right)$ there exists $\theta \in\left(2,2^{*}\right)$ such that $f(t) t-\theta F(t) \geq 0$ for $t \geq 0$, where $F(t)=\int_{0}^{t} f(s) d s$

$\left(\mathrm{f}_{6}\right)$ the function $f(t) / t$ is increasing for $t>0$.

The following theorem concerns the case in which $\left(\mathrm{a}_{2}\right)$ holds.

Theorem 1.1. Assume $N=3$ with $q>4$, or $N \geq 4$. If $\left(\mathrm{V}_{1}\right)-\left(\mathrm{V}_{2}\right),\left(\mathrm{a}_{1}\right)-\left(\mathrm{a}_{2}\right)$ and $\left(\mathrm{f}_{1}\right)-\left(\mathrm{f}_{6}\right)$ hold with $0<b<a<p$, then problem (1.1) has a ground state. 
On the contrary, considering the case in which $\left(a_{3}\right)$ holds, we also obtain a theorem.

Theorem 1.2. Assume $N=3$ with $q>4$, or $N \geq 4$. If $\left(\mathrm{V}_{1}\right),\left(\mathrm{V}_{3}\right),\left(\mathrm{a}_{1}\right)$, $\left(\mathrm{a}_{3}\right)$ and $\left(\mathrm{f}_{1}\right)-\left(\mathrm{f}_{6}\right)$ hold, then problem $(1.1)$ has a ground state for $\left\|V(x)-V_{\infty}\right\|_{N / 2}$ small enough.

The aim of this paper is to investigate equation (1.1) through the interaction of $V(x)$ and $a(x)$. Note that both $a(x) \leq a_{\infty}$ and $a(x) \geq a_{\infty}$ are considered with different conditions on $V(x)$ sufficient to guarantee the existence of ground states for (1.1). Because $V(x)$ and $a(x)$ are not radially symmetric functions, the usual variational techniques cannot be applied straightly due to the lack of compactness. The critical exponential growth makes the problem more complicated. Nevertheless, we can restore some compactness by establishing a global compactness lemma in the critical case and find a proper range of $c$ where the $(\mathrm{PS})_{c}$ condition holds for the associated functional. Then we obtain the existence of ground state solutions. In Theorem 1.1, the decay rate assumptions $\left(\mathrm{V}_{2}\right)$ and $\left(\mathrm{a}_{2}\right)$ are the key to energy estimate. In Theorem 1.2 , without any decay rate assumption, we still obtain a ground state solution by imposing that the $L^{N / 2}$ norm of $V(x)-V_{\infty}$ are suitably close to zero.

The outline of this paper is as follows: in Section 2, we establish some important lemmas. In Section 3, we prove Theorem 1.1. In Section 4, we prove Theorem 1.2.

\section{Notations.}

- $\|u\|_{s}:=\left(\int_{\mathbb{R}^{N}}|u|^{s} d x\right)^{1 / s}, 2 \leq s \leq \infty$.

- Let $H^{1}\left(\mathbb{R}^{N}\right)$ be the Hilbert space equipped with the norm

$$
\|u\|_{H^{1}}^{2}:=\int_{\mathbb{R}^{N}}\left(|\nabla u|^{2}+u^{2}\right) d x .
$$

- Let $D^{1,2}\left(\mathbb{R}^{N}\right):=\left\{u \in L^{2^{*}}\left(\mathbb{R}^{N}\right): \nabla u \in L^{2}\left(\mathbb{R}^{N}\right)\right\}$ be the Sobolev space equipped with the norm

$$
\|u\|_{D^{1,2}}^{2}:=\int_{\mathbb{R}^{N}}|\nabla u|^{2} d x .
$$

- $C$ denotes a universal positive constant.

- $B_{r}\left(x_{0}\right)$ denotes the open ball centered at $x_{0}$ with radius $r>0$.

- $S$ denotes the best Sobolev constant:

$$
S:=\inf _{u \in D^{1,2}\left(\mathbb{R}^{N}\right) \backslash\{0\}} \frac{\int_{\mathbb{R}^{N}}|\nabla u|^{2} d x}{\left(\int_{\mathbb{R}^{N}}|u|^{2^{*}} d x\right)^{2 / 2^{*}} .}
$$




\section{Preliminary lemmas}

In this section, we assume $\left(\mathrm{V}_{1}\right),\left(\mathrm{a}_{1}\right)$ and $\left(\mathrm{f}_{1}\right)-\left(\mathrm{f}_{6}\right)$ hold. Let

$$
H=\left\{u \in H^{1}\left(\mathbb{R}^{N}\right): \int_{\mathbb{R}^{N}} V(x)|u|^{2} d x<\infty\right\}
$$

be the Hilbert space equipped with the norm

$$
\|u\|^{2}=\int_{\mathbb{R}^{N}}\left(|\nabla u|^{2}+V(x)|u|^{2}\right) d x .
$$

From $\left(\mathrm{V}_{1}\right)$, we know that the embedding $H \hookrightarrow H^{1}\left(\mathbb{R}^{N}\right)$ is continuous. The functional associated with (1.1) is

$$
I(u)=\frac{1}{2}\|u\|^{2}-\int_{\mathbb{R}^{N}} a(x) F(u) \mathrm{d} x-\frac{1}{2^{*}} \int_{\mathbb{R}^{N}}|u|^{2^{*}} \mathrm{~d} x,
$$

where $u \in H$. It is easy to check that the functional $I: H \mapsto \mathbb{R}$ is of class $C^{1}$. Moreover, critical points of $I$ are weak solutions of (1.1). For simplicity, we may assume that $V_{\infty}=1$ and $a_{\infty}=1$ in this paper.

Lemma 2.1. There is a sequence $\left\{u_{n}\right\} \subset H$ such that $\left\{u_{n}\right\}$ is bounded in $H$, $I\left(u_{n}\right) \rightarrow c$ and $I^{\prime}\left(u_{n}\right) \rightarrow 0$. Moreover, $c \in\left(0,(1 / N) S^{N / 2}\right)$ for $N=3$ with $q>4$, or $N \geq 4$.

Proof. The conditions $\left(\mathrm{a}_{1}\right)$ and $\left(\mathrm{f}_{1}\right)-\left(\mathrm{f}_{3}\right)$ imply that for all $\varepsilon>0$, there exists $C(\varepsilon)>0$ such that

$$
|a(x) F(u)| \leq \varepsilon|u|^{2}+C(\varepsilon)|u|^{2^{*}} .
$$

In view of (2.2) and the embedding $H \hookrightarrow H^{1}\left(\mathbb{R}^{N}\right)$ is continuous, there holds

$$
\begin{aligned}
I(u) & \geq \frac{1}{2}\|u\|^{2}-\varepsilon \int_{\mathbb{R}^{N}} u^{2} \mathrm{~d} x-C(\varepsilon) \int_{\mathbb{R}^{N}}|u|^{2^{*}} d x-\frac{1}{2^{*}} \int_{\mathbb{R}^{N}}|u|^{2^{*}} d x \\
& \geq C\|u\|_{H^{1}}^{2}-C \int_{\mathbb{R}^{N}}|u|^{2^{*}} d x \geq C\|u\|^{2}-C \int_{\mathbb{R}^{N}}|u|^{2^{*}} d x .
\end{aligned}
$$

By Sobolev embedding theorem, there exists $r>0$ such that for $\|u\|=r$, $I(u) \geq \alpha>0$. From $\left(\mathrm{a}_{1}\right)$ and $\left(\mathrm{f}_{4}\right)$,

$$
I(u) \leq \frac{1}{2}\|u\|^{2}-\frac{1}{2^{*}} \int_{\mathbb{R}^{N}}|u|^{2^{*}} d x .
$$

Set $\varphi \in H$ such that $\varphi \neq 0$. Then $\lim _{t \rightarrow+\infty} I(t \varphi)=-\infty$. Thus, there exists $t_{0}>0$ such that $\left\|t_{0} \varphi\right\|>r$ and $I\left(t_{0} \varphi\right)<0$. We also have $I(0)=0$. Define

$$
c=\inf _{\gamma \in \Gamma} \max _{t \in[0,1]} I(\gamma(t)),
$$

where $\Gamma=\{\gamma \in C([0,1], H) ; \gamma(0)=0, I(\gamma(1))<0\}$. Then it follows from the mountain pass theorem in [3] that there is a sequence $\left\{u_{n}\right\} \subset H$ such that 
$I\left(u_{n}\right) \rightarrow c \geq \alpha$ and $I^{\prime}\left(u_{n}\right) \rightarrow 0$. On the other hand, by $\left(f_{5}\right)$,

$$
\begin{aligned}
c+o(1)\left\|u_{n}\right\| \geq\left(\frac{1}{2}-\frac{1}{\theta}\right)\left\|u_{n}\right\|^{2}+\int_{\mathbb{R}^{N}}\left(\frac{1}{\theta} a(x) f\left(u_{n}\right) u_{n}-a(x) F\left(u_{n}\right)\right) d x \\
\geq\left(\frac{1}{2}-\frac{1}{\theta}\right)\left\|u_{n}\right\|^{2},
\end{aligned}
$$

which implies that $\left\{u_{n}\right\}$ is bounded in $H$.

From $\left(\mathrm{a}_{1}\right)$, there exists $R>0$ such that $a(x) \geq 1 / 2$ for $|x| \geq R$. Choose $x_{0} \in \mathbb{R}^{N}$ and $r>0$ such that $B_{2 r}\left(x_{0}\right) \subset \mathbb{R}^{N} \backslash B_{R}(0)$. For $\varepsilon>0$, define the function $u_{\varepsilon}(x)=\psi(x) \varepsilon^{(N-2) / 4} /\left(\varepsilon+\left|x-x_{0}\right|^{2}\right)^{(N-2) / 2}$, where $\psi \in C_{0}^{\infty}\left(B_{2 r}\left(x_{0}\right)\right)$ such that $0 \leq \psi(x) \leq 1$ and $\psi(x)=1$ on $B_{r}\left(x_{0}\right)$. Note that $S$ is attained by the functions $\varepsilon^{(N-2) / 4} /\left(\varepsilon+\left|x-x_{0}\right|^{2}\right)^{(N-2) / 2}$. From [9], [28], we know that

$$
\begin{aligned}
\int_{\mathbb{R}^{N}}\left|\nabla u_{\varepsilon}\right|^{2} d x & =(N-2)^{2} \int_{\mathbb{R}^{N}} \frac{|x|^{2}}{\left(1+|x|^{2}\right)^{N}} d x+O\left(\varepsilon^{(N-2) / 2}\right) \\
& :=K_{1}+O\left(\varepsilon^{(N-2) / 2}\right), \\
\int_{\mathbb{R}^{N}}\left|u_{\varepsilon}\right|^{2^{*}} d x & =\int_{\mathbb{R}^{N}} \frac{1}{\left(1+|x|^{2}\right)^{N}} d x:=K_{2}+O\left(\varepsilon^{N / 2}\right)
\end{aligned}
$$

and

$$
\int_{\mathbb{R}^{N}}\left|u_{\varepsilon}\right|^{t} d x= \begin{cases}K \varepsilon^{(2 N-(N-2) t) / 4}, & t>N /(N-2), \\ K \varepsilon^{N / 4}|\ln \varepsilon|, & t=N /(N-2), \\ K \varepsilon^{t(N-2) / 4}, & t<N /(N-2),\end{cases}
$$

where $K_{1}, K_{2}, K$ are positive constants. Moreover, $S=K_{1} / K_{2}^{2 / 2^{*}}$. The definition of $c$ implies that $c \leq \sup _{t \geq 0} I\left(t u_{\varepsilon}\right)$. From $\left(\mathrm{V}_{1}\right),(2.4)$ and (2.6), we can choose $t^{\prime} \in(0,1)$ such that for $\varepsilon \in(0,1)$,

$$
\sup _{0 \leq t \leq t^{\prime}} I\left(t u_{\varepsilon}\right) \leq \sup _{0 \leq t \leq t^{\prime}} \frac{1}{2} t^{2}\left\|u_{\varepsilon}\right\|^{2} \leq \sup _{0 \leq t \leq t^{\prime}} C t^{2}\left\|u_{\varepsilon}\right\|_{H^{1}}^{2}<\frac{1}{N} S^{N / 2} .
$$

Thus, we only need to prove that $\sup _{t \geq t^{\prime}} I\left(t u_{\varepsilon}\right)<S^{N / 2} / N$. Define

$$
y(t):=\frac{1}{2} t^{2}\left\|u_{\varepsilon}\right\|^{2}-\frac{1}{2^{*}} t^{2^{*}} \int_{\mathbb{R}^{N}}\left|u_{\varepsilon}\right|^{2^{*}} d x .
$$

It is easy to check that $y(t)$ attains its maximum at

$$
t_{0}=\left(\frac{\left\|u_{\varepsilon}\right\|^{2}}{\left(\int_{\mathbb{R}^{N}}\left|u_{\varepsilon}\right|^{2^{*}} d x\right)}\right)^{(N-2) / 4} .
$$

Thus, by (2.4)-(2.6), we have

(2.8) $y\left(t_{0}\right)=\frac{1}{N}\left[\frac{\left\|u_{\varepsilon}\right\|^{2}}{\left(\int_{\mathbb{R}^{N}}\left|u_{\varepsilon}\right|^{2^{*}} d x\right)^{2 / 2^{*}}}\right]^{N / 2}=\frac{1}{N}[S+O(\varepsilon)]^{N / 2}=\frac{1}{N} S^{N / 2}+O(\varepsilon)$. 
By $\left(\mathrm{f}_{4}\right)$ and $a(x) \geq 1 / 2$ for $|x| \geq R$, there holds

$$
\sup _{t \geq t^{\prime}} I\left(t u_{\varepsilon}\right) \leq \sup _{t \geq 0} y(t)-C\left(t^{\prime}\right)^{q} \int_{\mathbb{R}^{N}}\left|u_{\varepsilon}\right|^{q} d x .
$$

For $N>4$, we derive from (2.6) and (2.8)-(2.9) that

$$
\sup _{t \geq t^{\prime}} I\left(t u_{\varepsilon}\right) \leq \frac{1}{N} S^{N / 2}+O(\varepsilon)-C \varepsilon^{(2 N-(N-2) q) / 4} .
$$

Observe that $(2 N-(N-2) q) / 4<1$. Then there exists $\varepsilon_{0} \in(0,1)$ such that for $\varepsilon \in\left(0, \varepsilon_{0}\right)$, there holds $\sup _{t>t^{\prime}} I\left(t u_{\varepsilon}\right)<S^{N / 2} / N$. Then $c<1 / N S^{N / 2}$ for $N>4$. Similar argument shows that $c<S^{N / 2} / N$ for $N=3$ with $q>4$, or $N=4$.

Define the functional $I^{\infty}$ :

$$
I^{\infty}(u)=\frac{1}{2} \int_{\mathbb{R}^{N}}\left(|\nabla u|^{2}+u^{2}\right) d x-\int_{\mathbb{R}^{N}} F(u) d x-\frac{1}{2^{*}} \int_{\mathbb{R}^{N}}|u|^{2^{*}} d x, \quad u \in H .
$$

LEMma 2.2. If $\left\{u_{n}\right\} \subset H$ is a sequence such that $\left\|u_{n}\right\|$ is bounded, $I\left(u_{n}\right) \rightarrow$ $c \in\left(0, S^{N / 2} / N\right)$ and $I^{\prime}\left(u_{n}\right) \rightarrow 0$, then there exists a subsequence of $\left\{u_{n}\right\}$, still denoted by $\left\{u_{n}\right\}$, an integer $k \in \mathbb{N} \cup\{0\}, w^{i} \in H$ for $1 \leq i \leq k$ such that

(a) $u_{n} \rightarrow u$ weakly in $H$ with $I^{\prime}(u)=0$,

(b) $w^{i} \neq 0$ and $I^{\infty^{\prime}}\left(w^{i}\right)=0$ for $1 \leq i \leq k$,

(c) $c=I(u)+\sum_{i=1}^{k} I^{\infty}\left(w^{i}\right)$,

where we agree that in the case $k=0$, the above holds without $w^{i}$.

Proof. From $\left\|u_{n}\right\|$ is bounded, we know that up to a subsequence, $u_{n} \rightarrow u$ weakly in $H$. It is easy to check that $I^{\prime}(u)=0$. Thus, (a) holds.

From Lemma 3.2 in [15], we know that for $s \in\left[2,2^{*}\right)$, there exists a subsequence $\left\{u_{n_{j}}\right\}$ such that for any $\varepsilon>0$, there exists $r_{\varepsilon}>0$ with

$$
\limsup _{j \rightarrow \infty} \int_{B_{j}(0) \backslash B_{r}(0)}\left|u_{n_{j}}\right|^{s} d x \leq \varepsilon
$$

for all $r \geq r_{\varepsilon}$. Choose $\eta \in C_{0}^{\infty}\left(B_{2}(0)\right)$ such that $0 \leq \eta(x) \leq 1$ and $\eta(x)=1$ on $B_{1}(0)$. Define $\bar{u}_{j}(x)=\eta(2|x| / j) u(x)$. It is easy to check that $\bar{u}_{j} \rightarrow u$ in $H$. Thus, similar to the proof of Lemma 3.5 in [15], we can prove that up to a subsequence,

$$
\begin{aligned}
\left\|u_{n}-\bar{u}_{n}\right\|^{2} & =\left\|u_{n}\right\|^{2}-\|u\|^{2}+o(1), \\
c-I(u) & =I\left(u_{n}-\bar{u}_{n}\right)+o(1)
\end{aligned}
$$

and

$$
I^{\prime}\left(u_{n}-\bar{u}_{n}\right)=o(1)
$$


Note that $u_{n}-\bar{u}_{n} \rightarrow 0$ weakly in $H$. Then by $\left(\mathrm{V}_{1}\right),\left(\mathrm{a}_{1}\right)$ and $(2.11)-(2.12)$, there holds

$$
\begin{gathered}
c-I(u)=I^{\infty}\left(u_{n}-\bar{u}_{n}\right)+o(1), \\
I^{\infty^{\prime}}\left(u_{n}-\bar{u}_{n}\right)=o(1) .
\end{gathered}
$$

Set $w_{n}^{1}=u_{n}-\bar{u}_{n}$. We will consider two cases.

Case 1. $\lim _{n \rightarrow \infty} \sup _{y \in \mathbb{R}^{N}} \int_{B_{1}(y)}\left|w_{n}^{1}\right|^{2} d x=0$.

The Lions Lemma in [28] implies that

$$
w_{n}^{1} \rightarrow 0 \text { in } L^{t}\left(\mathbb{R}^{N}\right) \text {, for all } t \in\left(2,2^{*}\right) .
$$

From $\left(\mathrm{a}_{1}\right)$ and $\left(\mathrm{f}_{1}\right)-\left(\mathrm{f}_{3}\right)$, for any $\varepsilon>0$, there exist $p \in\left(2,2^{*}\right)$ and $C(\varepsilon)>0$ such that

$$
|a(x) F(u)| \leq \varepsilon\left(|u|^{2}+|u|^{2^{*}}\right)+C(\varepsilon)|u|^{p} .
$$

Combining (2.13)-(2.16), there holds

$$
\begin{gathered}
c-I(u)=\frac{1}{2}\left\|w_{n}^{1}\right\|_{H^{1}}^{2}-\frac{1}{2^{*}} \int_{\mathbb{R}^{N}}\left|w_{n}^{1}\right|^{2^{*}} d x+o(1), \\
\left\|w_{n}^{1}\right\|_{H^{1}}^{2}-\int_{\mathbb{R}^{N}}\left|w_{n}^{1}\right|^{2^{*}} d x=o(1) .
\end{gathered}
$$

We may assume that $\left\|w_{n}^{1}\right\|_{H^{1}}^{2} \rightarrow \rho$. If $\rho>0$, then Sobolev embedding theorem implies that

$$
S \leq \frac{\left\|w_{n}^{1}\right\|_{H^{1}}^{2}}{\left(\int_{\mathbb{R}^{N}}\left|w_{n}^{1}\right|^{2^{*}} d x\right)^{2 / 2^{*}}},
$$

from which we derive that $\rho \geq S^{N / 2}$. Thus, $c-I(u)=\rho / N \geq S^{N / 2} / N$. By $I^{\prime}(u)=0$, we have $c-I(u) \leq c<S^{N / 2} / N$, a contradiction. Then $c=I(u)$.

Case 2. There exists $\gamma_{1}>0$ such that

$$
\lim _{n \rightarrow \infty} \sup _{y \in \mathbb{R}^{N}} \int_{B_{1}(y)}\left|w_{n}^{1}\right|^{2} d x \geq \gamma_{1}>0 .
$$

Then there exists $y_{n}^{1} \in \mathbb{R}^{N}$ such that

$$
\left|y_{n}^{1}\right| \rightarrow \infty \quad \text { and } \quad \int_{B_{1}\left(y_{n}^{1}\right)}\left|w_{n}^{1}\right|^{2} d x \geq \frac{\gamma_{1}}{2}>0
$$

from which we derive that $w_{n}^{1}\left(\cdot+y_{n}^{1}\right) \rightarrow w^{1} \neq 0$ weakly in $H$,

$$
\begin{gathered}
c-I(u)=I^{\infty}\left(w_{n}^{1}\left(\cdot+y_{n}^{1}\right)\right)+o(1), \\
I^{\infty^{\prime}}\left(w_{n}^{1}\left(\cdot+y_{n}^{1}\right)\right)=o(1) .
\end{gathered}
$$


Thus, we have $I^{\infty^{\prime}}\left(w^{1}\right)=0$. Similar to (2.11)-(2.12), we know that there exists $\left\{\bar{w}_{n}^{1}\right\} \subset H$ such that $\bar{w}_{n}^{1} \rightarrow w^{1}$ in $H$,

$$
\begin{gathered}
c-I(u)-I^{\infty}\left(w^{1}\right)+o(1)=I^{\infty}\left(w_{n}^{2}\right), \\
I^{\infty^{\prime}}\left(w_{n}^{2}\right)=o(1),
\end{gathered}
$$

where $w_{n}^{2}=w_{n}^{1}\left(\cdot+y_{n}^{1}\right)-\bar{w}_{n}^{1}$. We also have $\left\|w_{n}^{2}\right\|^{2}=\left\|w_{n}^{1}\right\|^{2}-\left\|w^{1}\right\|^{2}+o(1)$. Together with (2.10), there holds

$$
\left\|w_{n}^{2}\right\|^{2}=\left\|u_{n}\right\|^{2}-\|u\|^{2}-\left\|w^{1}\right\|^{2}+o(1) .
$$

Note that $w_{n}^{2} \rightarrow 0$ weakly in $H$. Then either

$$
\lim _{n \rightarrow \infty} \sup _{y \in \mathbb{R}^{N}} \int_{B_{1}(y)}\left|w_{n}^{2}\right|^{2} d x=0,
$$

or there exists $\gamma_{2}>0$ such that

$$
\lim _{n \rightarrow \infty} \sup _{y \in \mathbb{R}^{N}} \int_{B_{1}(y)}\left|w_{n}^{2}\right|^{2} d x \geq \gamma_{2}>0 .
$$

If (2.22) holds, similar to the argument of Case 1, we have $c=I(u)+I^{\infty}\left(w^{1}\right)$. So we may assume (2.23) holds. Continuing the process of Case 2, we obtain $\left\{w_{n}^{i}\right\} \subset H,\left\{y_{n}^{i}\right\} \subset \mathbb{R}^{N}$ and $\left\{\bar{w}_{n}^{i}\right\} \subset H, i \in \mathbb{N}$ such that $\left|y_{n}^{i}\right| \rightarrow \infty, w_{n}^{i}\left(\cdot+y_{n}^{i}\right) \rightarrow$ $w^{i} \neq 0$ weakly in $H, \bar{w}_{n}^{i} \rightarrow w^{i}$ in $H, I^{\infty^{\prime}}\left(w^{i}\right)=0$,

$$
\begin{gathered}
c-I(u)-\sum_{i=1}^{l} I^{\infty}\left(w^{i}\right)+o(1)=I^{\infty}\left(w_{n}^{l+1}\right), \\
I^{\infty^{\prime}}\left(w_{n}^{l+1}\right)=o(1)
\end{gathered}
$$

and

$$
\left\|w_{n}^{l+1}\right\|^{2}=\left\|u_{n}\right\|^{2}-\|u\|^{2}-\sum_{i=1}^{l}\left\|w^{i}\right\|^{2}+o(1),
$$

where $w_{n}^{l+1}=w_{n}^{l}\left(\cdot+y_{n}^{l}\right)-\bar{w}_{n}^{l}, l \in \mathbb{N}$. Standard argument shows that there exists $\beta>0$ such that if $I^{\infty^{\prime}}\left(w^{i}\right)=0$, then $\left\|w^{i}\right\|^{2} \geq \beta>0$ independent of $\beta$. Together with (2.26), we conclude that $w_{n}^{l+1} \rightarrow 0$ at some $l=k$. Thus, by (2.24), we have $c=I(u)+\sum_{i=1}^{k} I^{\infty}\left(w^{i}\right)$.

From Theorem 1.1 in [29], we know that there exists $u_{\infty} \in H$ such that $u_{\infty}$ is radial, $I^{\infty}\left(u_{\infty}\right)=m_{\infty}$ and $I^{\infty^{\prime}}\left(u_{\infty}\right)=0$, where $m_{\infty}=\inf \left\{I^{\infty}(u) ; u \in H\right.$, $\left.u \neq 0, I^{\infty^{\prime}}(u)=0\right\}$. Note that $u_{\infty}$ is not sign-changing. We may assume $u_{\infty} \geq 0$ in $H$. Then by the Maximum Principle, we get $u_{\infty}$ is positive. 
REMARK 2.3. Set $g(t)=I^{\infty}\left(t u_{\infty}\right)$, where $t \in(0, \infty)$. Observe that

$$
g^{\prime}(t)=t\left[\int_{\mathbb{R}^{N}}\left(\left|\nabla u_{\infty}\right|^{2}+u_{\infty}^{2}\right) d x-\int_{\mathbb{R}^{N}} \frac{f\left(t u_{\infty}\right)}{t u_{\infty}} u_{\infty}^{2} d x-t^{2^{*}-2} \int_{\mathbb{R}^{N}}|u|^{2^{*}} d x\right] .
$$

From $\left(\mathrm{f}_{6}\right)$, we can derive that $g(t)$ has a unique critical point corresponding to its maximum. Since $I^{\infty^{\prime}}\left(u_{\infty}\right)=0$, this critical point should be achieved at $t=1$.

The following lemma in [24] is standard.

Lemma 2.4. Let $N>2$. Then there is a constant $C=C(N)$ such that

$$
|u(x)| \leq \frac{C}{|x|^{(N-2) / 2}}\|u\|, \quad \text { for all } x \neq 0,
$$

for any $u \in H_{r}^{1}\left(\mathbb{R}^{N}\right)$.

Lemma 2.5. For any $\delta \in(0,1)$, there exists $C=C(\delta)>0$ such that

$$
u_{\infty}(x) \leq C e^{-(1-\delta)|x|} .
$$

Proof. From Lemma 2.3, we know that $u_{\infty}(x) \rightarrow 0$ as $|x| \rightarrow \infty$. Then by $\left(\mathrm{f}_{2}\right)$, for any $\delta>0$, there exists $R=R(\delta)>0$ such that for $|x| \geq R$, there holds $1-f\left(u_{\infty}\right) / u_{\infty}-u_{\infty}^{2^{*}-2} \geq(1-\delta)^{2}$. Thus, $-\Delta u_{\infty}+(1-\delta)^{2} u_{\infty} \leq 0$ for $|x| \geq R$ and there exists $M=M(\delta)>0$ such that $u_{\infty}(x) \leq M$ for $|x|=R$. Let $v(x)=M e^{-(1-\delta)(|x|-R)}$. Direct calculation shows that $-\Delta v+(1-\delta)^{2} v \geq 0$ for $x \neq 0$. By the Maximum Principle, we conclude that $u_{\infty}(x) \leq M e^{-(1-\delta)(|x|-R)}$ for $|x| \geq R$. Then Lemma 2.4 follows easily.

\section{Proof of Theorem 1.1}

Proof of Theorem 1.1. From Lemma 2.1, there is a sequence $\left\{u_{n}\right\} \subset H$ such that $\left\|u_{n}\right\|$ is bounded, $I\left(u_{n}\right) \rightarrow c \in\left(0, S^{N / 2} / N\right)$ and $I^{\prime}\left(u_{n}\right) \rightarrow 0$. We claim that $c<m_{\infty}$. Let $\beta=(1,0, \ldots, 0)$ be a fixed unit vector in $\mathbb{R}^{N}$. It follows from the definition of $c$ that $c \leq \sup _{t>0} I\left(t u_{\infty}(x-R \beta)\right)$. From $\left(\mathrm{V}_{1}\right),\left(\mathrm{a}_{1}\right)$ and $\left(\mathrm{f}_{4}\right)$, we know that there exists $t^{\prime} \in(0,1)$ such that

$$
\sup _{0 \leq t \leq t^{\prime}} I\left(t u_{\infty}(x-R \beta)\right) \leq \frac{1}{2}\left|t^{\prime}\right|^{2}\left\|u_{\infty}(x-R \beta)\right\|^{2} \leq C\left|t^{\prime}\right|^{2}\left\|u_{\infty}\right\|_{H^{!}}^{2}<m_{\infty}
$$

independent of $R>0$. From $\left(\mathrm{V}_{1}\right),\left(\mathrm{a}_{1}\right)$ and $\left(\mathrm{f}_{4}\right)$, we also have that there exists $t^{\prime \prime}>0$ such that

$$
\begin{aligned}
& \sup _{t \geq t^{\prime \prime}} I\left(t u_{\infty}(x-R \beta)\right) \\
& \quad \leq \sup _{t \geq t^{\prime \prime}}\left(\frac{1}{2} t^{2}\left\|u_{\infty}(x-R \beta)\right\|^{2}-\frac{1}{2^{*}} t^{2^{*}} \int_{\mathbb{R}^{N}}\left|u_{\infty}\right|^{2^{*}} d x\right) \\
& \quad \leq \sup _{t \geq t^{\prime \prime}}\left(C t^{2}\left\|u_{\infty}\right\|_{H^{1}}^{2}-\frac{1}{2^{*}} t^{2^{*}} \int_{\mathbb{R}^{N}}\left|u_{\infty}\right|^{2^{*}} d x\right)<m_{\infty}
\end{aligned}
$$

independent of $R>0$. 
Thus, we only need to prove that $\sup _{t^{\prime} \leq t \leq t^{\prime \prime}} I\left(t u_{\infty}(x-R \beta)\right)<m_{\infty}$. Observe that by $\left(\mathrm{V}_{2}\right)$ and $\left(\mathrm{a}_{2}\right)$,

$$
\begin{aligned}
I\left(t u_{\infty}\right. & (x-R \beta)) \\
= & I^{\infty}\left(t u_{\infty}(x-R \beta)\right)+\frac{1}{2} t^{2} \int_{\mathbb{R}^{N}}(V(x)-1)\left|u_{\infty}(x-R \beta)\right|^{2} d x \\
& \quad-\frac{1}{p} t^{p} \int_{\mathbb{R}^{N}}(a(x)-1)\left|u_{\infty}(x-R \beta)\right|^{p} d x \\
\leq & I^{\infty}\left(t u_{\infty}\right)-\frac{C_{1}}{2} t^{2} \int_{\mathbb{R}^{N}} e^{-b|x+R \beta|}\left|u_{\infty}\right|^{2} d x \\
& +\frac{C_{2}}{p} t^{p} \int_{\mathbb{R}^{N}} e^{-a|x+R \beta|}\left|u_{\infty}\right|^{p} d x .
\end{aligned}
$$

Lemma 2.4 implies that

$$
\int_{\mathbb{R}^{N}} e^{-a|x+R \beta|}\left|u_{\infty}\right|^{p} d x \leq \int_{\mathbb{R}^{N}} e^{-a R} e^{a|x|-p(1-\delta)|x|} d x .
$$

Choose $\delta \in(0,1-a / p)$. Then

$$
\int_{\mathbb{R}^{N}} e^{-a|x+R \beta|}\left|u_{\infty}\right|^{p} \mathrm{~d} x \leq C e^{-a R} .
$$

Note that

$$
\int_{\mathbb{R}^{N}} e^{-b|x+R \beta|}\left|u_{\infty}\right|^{2} \mathrm{~d} x \geq e^{-b R} \int_{|x| \leq 1} e^{-b|x|}\left|u_{\infty}\right|^{2} \mathrm{~d} x \geq C e^{-b R} .
$$

Thus, from Remark 2.1 and (3.3)-(3.5),

$$
\sup _{t^{\prime} \leq t \leq t^{\prime \prime}} I\left(t u_{\infty}(x-R \beta)\right) \leq m_{\infty}+C e^{-a R}-C e^{-b R} .
$$

By $0<b<a$, we can choose $R$ large enough such that $\sup _{t^{\prime}<t<t^{\prime \prime}} I\left(t u_{\infty}(x-R \beta)\right)<$ $m_{\infty}$. Thus, we have $c<m_{\infty}$. Lemma 2.2 implies that $I$ satisfies the PalaisSmale condition at $c \in\left(0, m_{\infty}\right)$. Then we have $u_{n} \rightarrow u$ in $H, I(u)=c$ and $I^{\prime}(u)=0$. Let

$$
m=\inf \left\{I(v) ; v \in H, v \neq 0, I^{\prime}(v)=0\right\} .
$$

Since $I^{\prime}(u)=0$, we have $0 \leq m \leq I(u)<m_{\infty}$. By the definition of $m$, there exists $\left\{v_{n}\right\} \subset H$ such that $v_{n} \neq 0, I\left(v_{n}\right) \rightarrow m$ and $I^{\prime}\left(v_{n}\right)=0$. Standard argument shows that there exists $\gamma>0$ such that $\left\|v_{n}\right\|^{2} \geq \gamma>0$ independent of $n$, which implies that $m>0$. Then $m \in\left(0, m_{\infty}\right)$. Since $I$ satisfies the Palais-Smale condition at $c \in\left(0, m_{\infty}\right)$, we have $v_{n} \rightarrow v$ in $H, I(v)=m$ and $I^{\prime}(v)=0$. 


\section{Proof of Theorem 1.2}

In this section, we may assume that meas $\left\{x \in \mathbb{R}^{N} ; a(x)>1\right\}>0$. Similar argument as in [18] can derive the following result.

Lemma 4.1. There exists $\gamma \in C([0,1], H)$ such that $\gamma(0)=0, I^{\infty}(\gamma(1))<0$, $u_{\infty} \in \gamma([0,1])$ and $\max _{t \in[0,1]} I^{\infty}(\gamma(t))=I^{\infty}\left(u_{\infty}\right)=m_{\infty}$. Moreover, $0 \notin \gamma((0,1])$.

Define the functional $J$ :

$$
J(u)=\frac{1}{2} \int_{\mathbb{R}^{N}}\left(|\nabla u|^{2}+u^{2}\right) d x-\int_{\mathbb{R}^{N}} a(x) F(u) \mathrm{d} x-\frac{1}{2^{*}} \int_{\mathbb{R}^{N}}|u|^{2^{*}} d x, \quad u \in H .
$$

Lemma 4.2. The functional $J$ admits a nontrivial critical point $w \in H$. Moreover, we have $J(w) \in\left(0, m_{\infty}\right)$ and $\|w\|_{H^{1}}^{2}<2 \theta m_{\infty} /(\theta-2)$.

Proof. Define

$$
c_{a}=\inf _{\gamma \in \Gamma} \max _{t \in[0,1]} J(\gamma(t)),
$$

where $\Gamma=\{\gamma \in C([0,1], H) ; \gamma(0)=0, J(\gamma(1))<0\}$. By Lemma 2.1, we can obtain a sequence $\left\{u_{n}\right\} \subset H$ such that $u_{n} \rightarrow w$ weakly in $H, J\left(u_{n}\right) \rightarrow c_{a} \in$ $\left(0,1 / N S^{N / 2}\right)$ and $J^{\prime}\left(u_{n}\right) \rightarrow 0$. Lemma 2.2 implies that $J$ satisfies the PalaisSmale condition at $c_{a} \in\left(0, m_{\infty}\right)$. Now we claim that $c_{a} \in\left(0, m_{\infty}\right)$. Lemma 4.1 implies that $\max _{t \in[0,1]} I^{\infty}(\gamma(t))=m_{\infty}$, where $\gamma \in C([0,1], H)$ such that $\gamma(0)=0$, $I^{\infty}(\gamma(1))<0$ and $0 \notin \gamma((0,1])$. From $\left(\mathrm{a}_{3}\right)$, we know that $J(\gamma(t))<I^{\infty}(\gamma(t))$ for all $t \in(0,1]$. Thus, the definition of $c_{a}$ implies that

$$
c_{a} \leq \max _{t \in[0,1]} J(\gamma(t))<\max _{t \in[0,1]} I^{\infty}(\gamma(t))=m_{\infty} .
$$

Then we have $u_{n} \rightarrow w$ in $H, J(w) \in\left(0, m_{\infty}\right)$ and $J^{\prime}(w)=0$. Similar to (2.3), we have $c_{a} \geq(1 / 2-1 / \theta)\|w\|_{H^{1}}^{2}$, from which we get $\|w\|_{H^{1}}^{2}<2 \theta m_{\infty} /(\theta-2)$.

REmark 4.3. Set $h(t)=J(t w)$, where $t \in(0, \infty)$. Similar to Remark 2.1, we know that $h(t)$ has a unique critical point at $t=1$ corresponding to its maximum.

Proof of Theorem 1.2. From Lemma 2.1, there is a sequence $\left\{u_{n}\right\} \subset H$ such that $\left\|u_{n}\right\|$ is bounded, $I\left(u_{n}\right) \rightarrow c \in\left(0, S^{N / 2} / N\right)$ and $I^{\prime}\left(u_{n}\right) \rightarrow 0$. We claim that $c<m_{\infty}$. Similar to the proof of Theorem 1.1, we know that there exist $t_{1} \in(0,1)$ and $t_{2}>1$ such that $\sup _{0 \leq t \leq t_{1}} I(t w)<m_{\infty}$ and $\sup _{t \geq t_{2}} I(t w)<m_{\infty}$. From $\left(\mathrm{V}_{3}\right)$,

$$
I(t w)=J(t w)+\frac{1}{2} t^{2} \int_{\mathbb{R}^{N}}(V(x)-1) w^{2} d x \leq J(t w)+\frac{1}{2} t^{2}\|w\|_{2^{*}}^{2}\|V(x)-1\|_{N / 2} .
$$

Thus, by Lemma 4.2 and Remark 4.1,

$$
\sup _{t_{1} \leq t \leq t_{2}} I(t w) \leq \sup _{t \geq 0} h(t)+C\|V(x)-1\|_{N / 2}
$$


which implies that $\sup _{t_{1} \leq t \leq t_{2}} I(t w)<m_{\infty}$ for $\|V(x)-1\|_{N / 2}$ small enough. The rest of the proof is similar to the proof of Theorem 1.1, we omit it here.

\section{REFERENCES}

[1] C.O. Alves, M.A.S. Souto And M. Montenegro, Existence of a ground state solution for a nonlinear scalar field equation with critical growth, Calc. Var. Partial Differential Equations 43 (2012), 537-554.

[2] A. Ambrosetti, V. Felli and A. Malchiodi, Ground states of nonlinear Schrödinger equations with potentials vanishing at infinity, J. Eur. Math. Soc. 7 (2005), 117-144.

[3] A. Ambrosetti And P.H. Rabinowitz, Dual variational methods in critical point theory and applications, J. Funct. Anal. 14 (1973), 349-381.

[4] T. BARTSCH AND Z.-Q. WANG, Existence and multiplicity results for some superlinear elliptic problems on $\mathbb{R}^{N}$, Comm. Partial Differential Equations 20 (1995), 1725-1741.

[5] _ Multiple positive solutions for a nonlinear Schrödinger equations, Z. Angew. Math. Phys. 51 (2000), 366-384.

[6] T. Bartsch, A. Pankov and Z.-Q. Wang, Nonlinear Schrödinger equations with steep potential well, Comm. Contemp. Math. 4 (2001), 549-569.

[7] H. Berestycki, T. Gallouët And O. Kavian, Equations de champs scalaires euclidiens non linéaire dans le plan, C.R. Acad. Sci. Paris Sér. I Math. 297 (1983), 307-310.

[8] H. Berestycki And P. L. Lions, Nonlinear scalar field equations I. Existence of a ground state, Arch. Ration. Mech. Anal. 82 (1983), 313-346.

[9] H. Brezis and L. Nirenberg, Positive solutions of nonlinear elliptic equations involving critical Sobolev exponents, Comm. Pure Appl. Math. 36 (1983), 437-477.

[10] M. Clapp And Y.H. Ding, Positive solutions of a Schrödinger equation with critical nonlinearity, Z. Angew. Math. Phys. 55 (2004), 592-605.

[11] J. Chabrowski ANd A. Szulkin, On a semilinear Schrödinger equation with critical Sobolev exponent, Proc. Amer. Math. Soc. 130 (2001), 85-93.

[12] J. Chabrowski And J. YANG, Existence theorems for the Schrödinger equation involving a critical Sobolev exponent, Z. Angew. Math. Phys. 49 (1998), 276-293.

[13] _ On Schrödinger equation with periodic potential and critical Sobolev exponent, Topol. Methods Nonlinear Anal. 12 (1998), 245-261.

[14] V. Coti Zelati and P.H. Rabinowitz, Homoclinic type solutions for a semilinear elliptic PDE on $\mathbb{R}^{N}$, Comm. Pure Appl. Math. XIV (1992), 1217-1269.

[15] Y.H. Ding AND F.H. Lin, Solutions of perturbed Schrödinger equations with critical nonlinearity, Calc. Var. Partial Differential Equations 30 (2007), 231-249.

[16] Y.H. Ding And K. TANaKa, Multiplicity of positive solutions of a nonlinear Schrödinger equation, Manuscripta Math. 112 (2003), 109-135.

[17] L. Jeanjean, On the existence of bounded Palais-Smale sequences and application to a Landesman-Lazer-type problem set on $\mathbb{R}^{N}$, Proc. Roy. Soc. Edinburgh 129 (1999), 787-809.

[18] L. Jeanjean and K. TANaKa, A positive solution for asymptotically linear elliptic problem on $\mathbb{R}^{N}$ autonomous at infinity, ESAIM Control Optim. Calc. Var. 7 (2002), 597-614.

[19] _ A positive solution for a nonlinear Schrödinger equation on $\mathbb{R}^{N}$, Indiana Univ. Math. J., 54 (2005), 443-464.

[20] Y.Q. Li, Z.-Q. Wang AND J. Zeng, Ground states of nonlinear Schrödinger equations with potentials, Ann. Inst. H. Poincaré Anal. Non Linéaire 23 (2006), 829-837. 
[21] C. LiU, Z. WANG AND H. Zhou, Asymptotically linear Schrödinger equations with potential vanishing at infinity, J. Differential Equations 245 (2008), 201-222.

[22] P.H. Rabinowitz, On a class of nonlinear Schrödinger equations, Z. Angew. Math. Phys. 43 (1992), 270-291.

[23] M. Schechter AND W. Zou, Weak linking theorems and Schrödinger equations with critical Sobolev exponent, ESAIM: Control, Optimisation and Calculus of Variations 9 (2003), 601-619.

[24] W.A. Strauss, Existence of solitary waves in higher dimensions, Comm. Math. Phys. 55 (1977), 149-162.

[25] A. Szulkin And T. Weth, Ground state solutions for some indefinite variational problems, J. Funct. Anal. 257 (2009), 3802-3822.

[26] F.A. VAN HEERden AND Z.-Q. WANG, Schrödinger type equations with asymptotically linear nonlinearities, Differential Integral Equations, 16 (2003), 257-280.

[27] F.A. VAN HEERDEN, Multiple solutions for a Schrödinger type equation with an asymptotically linear term, Nonlinear Anal. 55 (2003), 739-758.

[28] M. Willem, Minimax Theorems, Birkhäuser, Boston (1996).

[29] J. ZHANG, On ground state solutions for quasilinear elliptic equations with a general nonlinearity in the critical growth, J. Math. Anal. Appl. 401 (2013), 232-241.

[30] J. Zhang And W. Zou, The critical case for a Berestycki-Lions theorem, Sci. China Math. 57 (2014), 541-555.

[31] J.J. Zhang And W. Zou, A Berestycki-Lions theorem revisited, Commun. Contemp. Math. 14 (2012), 125-133.

JIAN ZHANG

College of Science

China University of Petroleum

Qingdao 266580

Shandong, P.R. China

E-mail address: zjianmath@163.com 